\title{
Comparando periódicos pela medida da dispersão de seu impacto
}

Guido Rummler

\author{
Professor Titular da Universidade Estadual de \\ Feira de Santana - UEFS.
}

O presente estudo tem por objetivo demonstrar a aplicação de um algoritmo no dimensionamento da dispersão do impacto de periódicos sobre um grupo de publicações referenciais. Como objeto de exemplificação foram utilizados 14 periódicos nacionais de enfermagem, que foram então classificados de acordo com $o$ correspondente índice de dispersão segmentar - IDS, considerando os diversos níveis de distribuição das unidades impactadas. Nesse exemplo, reunindo periódicos marcados por expressivas diferenças quanto ao número de citações recebidas no período considerado, foram verificados valores do IDS de 0,005 a 4,810. A dispersão de impacto é interpretada como representação de uma condição potencial para a difusão da unidade citada.

Palavras-chave: Periódicos; Dispersão; Difusão; Impacto; Bibliometria; Informetria; Enfermagem.

\section{Comparing journals by the measure of its impact dispersion.}

The present study aims at demonstrating the application of an algorithm to measure the impact dispersion of journals over a referential publication group. Fourteen national Brazilian? nursing journals were used as the example object. They were classified according to the correspondent index of segmental dispersion - ISD, considering different distribution levels of the impacted units. In this example, which incorporate journals with expressive differences as for the number of received citations, ISD varied from 0.005 to 4.810 . The impact dispersion is interpreted as a condition that involves the continued diffusion of the cited unity. 
Keywords: Journals; Impact; Dispersion; Diffusion; Bibliometrics; Informetrics; Nursing.

Recebido em 25.10.2006 Aceito em 09.04.2007

\section{Introdução}

Diversos são os indicadores apontados na literatura que contribuem para a avaliação de periódicos científicos, seja quantitativa ou qualitativamente. Dentre esses indicadores possivelmente o de maior repercussão seja o Fator de Impacto - FI - do Institut for Scientific Information - ISI, cujo cálculo baseia-se no número de citações recebidas por uma revista em um conjunto de periódicos de referência, correspondente a um intervalo particular de tempo (STREHL, 2005; THOMSON, 2006).

Diversos outros indicadores do âmbito da Bibliometrica $e$ Cientometria (ou Cienciometria) dentre os enumerados e definidos em Vinkler (1988), Spinak (1998), Braun e Schubert (1997), Sen (1999), Glänzel e Moed (2002) e Portal (2005) requerem também análise de citações. Assim, dados colhidos por meio desta, e matematicamente operacionalizados, permitem dimensionar fenômenos específicos, tais como: atratividade, afinidade, imediatividade (ou imediatez), isolamento, acoplamento, obsolescência, popularidade etc. das respectivas unidades de análise.

Em trabalho recente (RUMMLER, 2006) foi desenvolvido um indicador com aplicabilidade à medição da dispersão literária, seja de autores, publicações temas, etc., que, utilizando dados obtidos por meio da análise de citações, permite dimensionar a dispersão de impacto dessas unidades, segundo níveis de sua distribuição (por artigo, fascículo, periódico, áreas de conhecimento, geográficas, etc.), e por isso denominado de Índice de Dispersão Segmentar - IDS.

Este aspecto da dispersão nivelar não é levado em conta, mesmo em indicadores da difusão de periódicos de proposição mais recente, como a de Rowlands (2002) e Frandsen (2005), que tem proximidade com o mesmo princípio de medida do Fator de Impacto do ISI. Outros autores, a exemplo de Kortelainen (2001), fazem avaliações da difusão de periódicos por analise do cruzamento dois-a-dois de parâmetros como ano e número de artigos ou periódicos citantes com o número de citações ao periódico alvo.

Assim, o presente trabalho tem por objetivo exemplificar, demonstrar e analisar uma das possibilidades de aplicação do acima referido IDS, que é a hierarquizar periódicos segundo a dispersão de seu impacto.

Vale mencionar que a dispersão de um objeto de informação, por exemplo, constitui um estágio de sua socialização, e a medida da dispersão de impacto um indicativo da sua abrangência. 


\section{Procedimentos}

O Índice de Dispersão Segmentar - IDS -, aplicado como indicador da dispersão do impacto de uma unidade de análise, constitui uma medida que se expressa em relação a um conjunto referencial de periódicos no qual é computada a quantidade de referências àquela unidade. Sendo, para efeito desse estudo, utilizados como unidade de análise de periódicos científicos de enfermagem editados no Brasil, foi considerado apropriado estabelecer como conjunto referencial às 22 unidades listadas no Portal de Revistas de Enfermagem (2006).

Após buscas, eletrônica e física, realizadas para obtenção dos fascículos correspondentes ao ano 2003, e a exclusão dos títulos sem edição ou edição incompleta do referido ano, foi possível o acesso a um elenco de 13 periódicos. A esse elenco foi acrescido outro periódico em formato eletrônico, ainda não constante da listagem daquele portal. Esse conjunto de 14 periódicos constituiu um acervo de 52 fascículos contendo 641 artigos. Sua identificação pelo título e volume das revistas, com a respectiva quantidade de fascículos e número de artigos neles publicados corresponde a: Acta Paulista de Enfermagem, v. 16 (4 e 42), Cogitare Enfermagem, v. 8 (2 e 20), Escola Anna Nery Revista de Enfermagem, v.7 (3 e 39), Nursing, v. 6 (8 e 40), Online Brazilian Journal of Nursing $O B J N$, v. 2 (3 e 24), Revista Eletrônica de Enfermagem, v. 5 (2 e 25), REME - Revista Mineira de Enfermagem, v. 7 (2 e 25), Revista Brasileira de Enfermagem, v.56 (5 e 105), Revista da Escola de Enfermagem da USP, v. 37 (4 e 47), Revista de Enfermagem da UERJ, v. 11 (3 e 49), Revista Gaúcha de Enfermagem, v. 24 (3 e 33), Revista Latino-americana de Enfermagem, v. 11 (6 e 102), Revista Paulista de Enfermagem, v. 22 (3 e 29), Texto e Contexto - Enfermagem, v. 12 (4 e 69).

Com base nos 641 artigos constituintes do conjunto referencial foram estabelecidos os escores respectivos a cada um daqueles periódicos quanto ao total $r$ de referências encontradas, número a de artigos que citam o periódico em foco, número $f$ de fascículos em que se encontram esses artigos e número $p$ de periódicos que publicaram no ano 2003 algum artigo que referencia o periódico em questão. $O$ índice de dispersão segmentar referente a cada periódico foi calculado de acordo com a expressão geral do algoritmo apresentado em artigo antecedente de Rummler (2006, p. 67):que na presente aplicação, abrangendo $k=3$ segmentos de distribuição (referência em artigo, artigo em fascículo, e, fascículo em periódico), pode assim ser representada:

$$
\operatorname{IDS}_{3 ; i}=\frac{\mathrm{p}_{\mathrm{i}} \cdot \mathrm{f}_{\mathrm{i}}}{\mathrm{P}^{2}}+\frac{\mathrm{f}_{\mathrm{i}} \cdot \mathrm{a}_{\mathrm{i}}}{\mathrm{F}^{2}}+\frac{\mathrm{a}_{\mathrm{i}} \cdot \mathrm{r}_{\mathrm{i}}}{\mathrm{A}^{2}}
$$

onde as constantes $\mathrm{P}, \mathrm{F}$ e $\mathrm{A}$ correspondem, respectivamente, ao número de periódicos que compõem o conjunto referencial $(P=14)$, o número total de fascículos $(F=52)$ e o total de artigos analisados $(A=641)$, e "i", o número identificador do periódico focalizado. 
Como as 3 parcelas que compõem o IDS constituem, respectivamente, os coeficientes $\mathrm{Ca}$ (do nível macro de distribuição), Ce (do nível meso) e Ci (do nível micro), pode-se, para simplificar sua apresentação, assim expressá-lo:

$\mathrm{IDS}_{3}=\mathrm{Ca}+\mathrm{Ce}+\mathrm{Ci}$.

Após o cálculo dos 14 índices de dispersão, foi estabelecida uma classificação dos periódicos de acordo com o ordenamento decrescente dos valores correspondentes a esse indicador. À tabela que mostra esse ordenamento dos periódicos e respectivos índices foram acrescentados, para fins de discussão dos resultados, os valores correspondentes às variáveis que integram o índice. Foi constituída ainda uma segunda tabela que permite visualizar o posto de classificação ocupado por cada periódico, quando este é estabelecido em função dos escores p, f, a, r e do número $L$ (que indica a longevidade do periódico pelo número do volume que foi editado em 2003).

Em etapa subseqüente foi avaliada a proporção (\%) com que cada coeficiente, respectivo a cada nível de distribuição, participa no valor final do IDS, mediante as seguintes operações, aplicadas a cada periódico, respectivas aos níveis da macro, meso e micro-distribuição:

Os limites de variação e a mediana dos 14 valores obtidos para cada uma dessas 3 expressões, foram destacados para caracterizar a contribuição relativa de cada nível de distribuição na constituição do IDS. $\mathrm{E}$, com base no que representam as relações entre os parâmetros respectivos a cada segmento de distribuição, foi inferida a natureza desse indicador. Para verificar se ordenamentos classificatórios estabelecidos de acordo com os diferentes parâmetros avaliados divergem significativamente, ou para testar a existência de correlação entre os mesmos, foram aplicados o Teste de Sinais de Wilcoxon e o Coeficiente Rô de Spearman, respectivamente, utilizando o Programa SPSS INC. (1998) e assumindo como nível de significância $p \leq 0,05$.

\section{Resultados}

A TAB. 1 identifica as unidades de análise utilizadas nesse estudo, reúne os dados globais apurados a partir do conjunto referencial, que correspondem a cada periódico, assim como o respectivo valor do IDS, aqui representando dispersão de impacto - DI -. Essa tabela mostra a ocorrência de variações do IDS compreendidas entre o maior valor obtido, de 4,810, e o menor, de 0,005. Esses extremos correspondem às situações em que periódicos foram citados por todos do conjunto ou por apenas um dos componentes do conjunto referencial, respectivamente. Da mesma forma correspondem extremos em que apenas um, ou mesmo quarenta fascículos, contém artigos que citam um mesmo periódico. A nível de artigos que citam determinado periódico, encontram-se variações entre 1 e 132, enquanto o número de referências recebidas por uma revista varia de 2 a 233. 
TABELA 1 - Escores correspondentes ao número de revistas (p) de enfermagem nas quais se encontram citações ao periódico avaliado, número de fascículos ( $f$ ) pelos quais encontra-se distribuído o total de artigos (a) e o total de referências ( $r$ ) a cada uma das 14 unidades que constituem o conjunto analizado. IDS é o Indice de Dispersão Segmentar utilizado como indicador da dispersão do impacto dos periódicos

\begin{tabular}{l|c|c|c|c|c}
\hline \multicolumn{1}{c|}{ PERIÓDICO* } & p & f & a & r & IDS \\
\hline 1. Revista Brasileira de Enfermagem & 14 & 40 & 132 & 233 & 4,810 \\
\hline 2. Revista da Escola de Enfermagem da USP & 13 & 40 & 69 & 127 & 3,695 \\
\hline 3. Revista Latino Americana de Enfermagem & 14 & 37 & 67 & 167 & 3,587 \\
\hline 4. Texto \& Contexto - Enfermagem & 12 & 31 & 72 & 105 & 2,741 \\
\hline 5. Acta Paulista de Enfermagem & 13 & 28 & 47 & 60 & 2,351 \\
\hline 6. Nursing (São Paulo) & 12 & 25 & 39 & 51 & 1,897 \\
\hline 7. Revista de Enfermagem UERJ & 12 & 23 & 32 & 38 & 1,683 \\
\hline 8. Revista Gaúcha de Enfermagem & 12 & 22 & 30 & 35 & 1,594 \\
\hline 9. Revista Paulista de Enfermagem & 11 & 22 & 25 & 28 & 1,440 \\
\hline 10. Escola Anna Nery Revista de Enfermagem & 9 & 13 & 32 & 36 & 0,757 \\
\hline 11. Cogitare Enfermagem & 7 & 11 & 12 & 21 & 0,443 \\
\hline 12. REME - Revista Mineira de Enfermagem & 3 & 3 & 3 & 3 & 0,049 \\
\hline 13. OBJN - Online Brazilian Journal of Nursing & 1 & 3 & 3 & 6 & 0,023 \\
\hline 14. Revista Eletrônica de Enfermagem & 1 & 1 & 1 & 2 & 0,005 \\
\hline * Por ordem de classificacão quanto ao IDS & & & &
\end{tabular}

* Por ordem de classificação quanto ao IDS

As posições classificatórias ocupadas por cada periódico com relação ao valor do IDS e $p, f$, a e $r$, que constam da TAB. 2, indicam que, exceto com relação ao periódico que apresenta maior IDS, em todos os demais ocorrem divergências quanto a postos que lhes correspondem em classificações estabelecidas quanto aos outros parâmetros.

Assim por exemplo, o periódico com o segundo melhor IDS tem essa mesma posição com relação ao número de revistas em que é citado, é o terceiro quanto ao número total de referências recebidas e número de artigos que o citam, e se iguala ao primeiro colocado quanto ao número de fascículos em que constam esses artigos.

Verifica-se ainda na TAB. 2 que a posição classificatória ocupada por um periódico quanto ao IDS, pode não ser igual a nenhuma das que the corresponde com relação aos demais parâmetros, sendo intermediária (periódico 3) ou estando fora do intervalo de ordenamento relativo às demais classificações (periódico 13). Tais questões são alvo da discussão a seguir apresentada e para a qual foi também incluída nessa tabela indicação referente à longevidade (L) dos periódicos. Também quanto a esse parâmetro verificam-se alternâncias quanto aos respectivos ordenamentos classificatórios. Entretanto, apenas as diferenças estabelecidas pela classificação por p e f têm significância estatística, onde 
a primeira diverge de todas as outras e a segunda só não diverge do ordenamento em função de $L$ por outro lado, cada um dos nove parâmetros levantados (incluindo os da TAB. 3) mantém correlação estatística.

TABELA 2 - Posições classificatórias ocupadas por periódicos de enfermagem, de acordo com o valor do IDS, $\mathrm{p}$ (número de revistas que é citado), $\mathrm{f}$ (número de fascículos em que há artigos que referenciam o periódico), a (número de artigos que citam o periódico), $r$ (número total de referências recebidas pelo periódico) e L (longevidade do periódico)

\begin{tabular}{|c|c|c|c|c|c|c|}
\hline \multirow{2}{*}{ Periódico } & \multicolumn{6}{|c|}{ Indicador } \\
\hline & IDS & $r$ & $p^{* \star}$ & 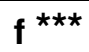 & a & $\mathbf{L}$ \\
\hline 1 * & $1^{\underline{a}}$ & $1^{\underline{a}}$ & $1^{\underline{a}}$ & $1^{\underline{a}}$ & $1^{\underline{a}}$ & $1^{\underline{a}}$ \\
\hline 2 & $2^{a}$ & $3^{\underline{a}}$ & $2^{\mathrm{a}}$ & $1^{\mathrm{a}}$ & $3^{a}$ & $2^{\underline{a}}$ \\
\hline 3 & $3^{\frac{a}{a}}$ & $2^{\underline{a}}$ & $1^{\underline{a}}$ & $2^{\mathrm{a}}$ & $4^{\mathrm{a}}$ & $7^{\mathrm{a}}$ \\
\hline 4 & $4^{\underline{a}}$ & $4^{\underline{a}}$ & $3^{\mathrm{a}}$ & $3^{\mathrm{a}}$ & $2^{\underline{a}}$ & $6^{\underline{a}}$ \\
\hline 5 & $5^{\underline{a}}$ & $5^{\underline{a}}$ & $2^{\underline{a}}$ & $4^{\mathrm{a}}$ & $5^{a}$ & $5^{\underline{a}}$ \\
\hline 6 & $6^{\underline{a}}$ & $6^{\underline{a}}$ & $3^{\underline{a}}$ & $5^{\underline{a}}$ & $6^{a}$ & $10^{\underline{a}}$ \\
\hline 7 & $7^{\mathrm{a}}$ & $7^{\underline{a}}$ & $3^{\underline{a}}$ & $6^{\mathrm{a}}$ & $7^{a}$ & $7^{\underline{a}}$ \\
\hline 8 & $8^{\mathrm{a}}$ & $9^{a}$ & $3^{\mathrm{a}}$ & $7^{\mathrm{a}}$ & $8^{a}$ & $3^{\mathrm{a}}$ \\
\hline 9 & $9 \underline{a}$ & $10^{\underline{a}}$ & $4^{\mathrm{a}}$ & $7^{\underline{a}}$ & $9^{a}$ & $4^{\mathrm{a}}$ \\
\hline 10 & $10^{\underline{a}}$ & $8^{a}$ & $5^{\underline{a}}$ & $9^{\underline{a}}$ & $10^{\underline{a}}$ & $9^{\underline{a}}$ \\
\hline 11 & $11^{\underline{a}}$ & $11^{\mathrm{a}}$ & $6^{\underline{a}}$ & $8^{\underline{a}}$ & $11^{\mathrm{a}}$ & $8^{\underline{a}}$ \\
\hline 12 & $12^{\mathrm{a}}$ & $13^{\underline{a}}$ & $7^{\mathrm{a}}$ & $10^{\mathrm{a}}$ & $12^{\underline{a}}$ & $9^{\underline{a}}$ \\
\hline 13 & $13^{\mathrm{a}}$ & $12^{\mathrm{a}}$ & $8^{\mathrm{a}}$ & $10^{\mathrm{a}}$ & $12^{\mathrm{a}}$ & $12^{\mathrm{a}}$ \\
\hline 14 & $14^{\underline{a}}$ & $14^{\underline{a}}$ & $8^{\underline{a}}$ & $11^{\mathrm{a}}$ & $13^{\underline{a}}$ & $11^{\mathrm{a}}$ \\
\hline
\end{tabular}

* número identificador do periódico de acordo com o que lhe corresponde na Tabela 1.

** diferença significativa $(p \leq 0,05)$ frente aos demais ordenamentos classificatórios.

*** diverge significativamente $(p \leq 0,05)$ de IDS, $r, p, a$.

Quanto aos dados que permitem avaliar a participação proporcional dos coeficientes respectivos a cada nível de distribuição, no valor calculado do IDS, a TAB. 3 indica as seguintes variações: para o coeficiente relativo ao nível macro de distribuição valores entre $58,5 \%$ a $100 \%$, com mediana de $82,2 \%$; para o coeficiente relativo ao nível meso de distribuição, valores entre zero e $40 \%$, com mediana de $17,6 \%$, enquanto o coeficiente respectivo à micro-distribuição varia de zero a $1,6 \%$, com mediana de $0,25 \%$. 
TABELA 3 - Contribuição relativa (\%) dos coeficientes de cada nível de distribuição no cálculo do índice de dispesão segmentar (IDS) e respectivos postos classificatórios correspondentes aos 14 periódicos de enfermagem

\begin{tabular}{|c|c|c|c|c|c|c|c|c|}
\hline \multirow{2}{*}{ Periódico * } & \multicolumn{2}{|c|}{ IDS } & \multicolumn{2}{|c|}{$\mathrm{Ca}$ ** } & \multicolumn{2}{|c|}{$\mathrm{Ce}^{* \star}$} & \multicolumn{2}{|c|}{$C i^{\star \star}$} \\
\hline & IDS & $P C^{* \star *}$ & $\%$ & $P C^{* \star \star}$ & $\%$ & $P C^{\star \star \star}$ & $\%$ & $P C^{\star \star \star}$ \\
\hline 1 & 4,810 & 1 & 58,5 & 1 & 40,0 & 1 & 1,6 & 1 \\
\hline 2 & 3,695 & 2 & 71,8 & 4 & 27,6 & 4 & 0,6 & 3 \\
\hline 3 & 3,587 & 3 & 73,7 & 2 & 25,6 & 2 & 0,7 & 4 \\
\hline 4 & 2,741 & 4 & 68,9 & 3 & 30,1 & 3 & 0,7 & 2 \\
\hline 5 & 2,351 & 5 & 79,0 & 5 & 20,7 & 5 & 0,3 & 10 \\
\hline 6 & 1,897 & 6 & 80,7 & 10 & 19,0 & 10 & 0,3 & 5 \\
\hline 7 & 1,683 & 7 & 83,7 & 6 & 16,2 & 6 & 0,1 & 6 \\
\hline 8 & 1,594 & 8 & 84,5 & 7 & 15,3 & 7 & 0,2 & 8 \\
\hline 9 & 1,440 & 9 & 85,8 & 8 & 14,1 & 8 & 0,1 & 11 \\
\hline 10 & 0,757 & 10 & 79,3 & 9 & 20,3 & 9 & 0,4 & 7 \\
\hline 11 & 0,443 & 11 & 88,7 & 13 & 11,1 & 13 & 0,2 & 9 \\
\hline 12 & 0,049 & 12 & 93,9 & 11 & 6,1 & 11 & 0,1 & 12 \\
\hline 13 & 0,023 & 13 & 87,0 & 12 & 13 & 12 & - & - \\
\hline 14 & 0,005 & 14 & 100 & 14 & - & - & - & - \\
\hline Mediana & 1,638 & & 82,2 & & 17,6 & & 0,25 & \\
\hline
\end{tabular}

* Mesma identificação numérica da Tabela 1.

${ }^{* *} \mathrm{Ca}, \mathrm{Ce}, \mathrm{Ci}$ - coeficientes respectivos aos níveis de distribuição macro, meso e micro.

${ }^{* * *}$ PC - Posto de Classificação.

\section{Discussão}

Considere-se que o objetivo precípuo do presente trabalho é o de demonstrar uma das possíveis aplicações do IDS, no caso, a representação e avaliação da dispersão do impacto de periódicos. Porém, em se tratando de um indicador relativo, há que se considerar observações de Vinkler (2003) e de Van Raan (2004) quanto ao fato de que a padronização do conjunto de referência (acervo) em relação ao qual é calculado um indicador relativo, como é o caso do IDS, constitui passo crucial do processo de avaliação. Assim, ao serem utilizados periódicos brasileiros da área de enfermagem como alvo dessa demonstração, foi instituído, a priori, como conjunto de referência, os periódicos listados no Portal de Revistas de Enfermagem (2006) que oferece informações sobre possível acesso e/ou localização dessas unidades. Foi também considerada a premissa de que esse portal identifica as revistas que concentram a produção científica brasileira da área de enfermagem, assim como concentra referências a essa produção. Dessa forma, é possível que, embora restrito, o conjunto assim originado constitui adequado elenco de referência que atende peculiaridades desse campo. É pertinente também mencionar que é intrínseco ao IDS relativizar sua medida ao número de 
unidades de referência que compõem cada nível de distribuição, aspecto detalhado no já mencionado trabalho que mostra sua modelagem, não sendo, portanto, o tamanho da coleção de referência um fator crítico para aplicação desse indicador. Por outro lado, deve-se considerar também que, diferente dos critérios de indexação pelos quais é constituída, por exemplo, a coleção de periódicos referenciais no cálculo do Fator de Impacto - FI do ISI (THOMSON SCIENTIFIC, 2005), a inclusão, ou não inclusão, de periódicos nacionais de enfermagem não reporta aqui juízo de qualidade. Por outro lado, convém que para uma avaliação que pretenda testar o comportamento de um indicador, seja esta aplicada em um conjunto heterogêneo de objetos de avaliação, aspecto contemplado pelo conjunto escolhido.

No presente estudo tem-se ainda, atendendo a definição de unidade de análise de Singlenton (1970), citada por Frota (1998, p. 262) e a diferenciação entre esta e o conceito de variável, destacada por McGrath (1996), que cada um dos 14 periódicos elencados constitui ao mesmo tempo unidade de análise e um elemento de conjunto referencial. No primeiro caso as variáveis $p, f, a$ e $r$ caracterizam o periódico. No segundo, o periódico fornece medida dessas variáveis referente aos demais e de si mesmo, vez que nesta análise não é descartada a autocitação. A influência da auto-citação é, por outro lado, um aspecto que se pretende analisar mais amiúde em estudo vindouro.

Quanto aos valores obtidos para o IDS, ou demais parâmetros apresentados na TAB. 1, verifica-se que os 14 periódicos compõem um conjunto em que há expressivas diferenças entre unidades constituintes. Conjecturas a respeito de um variado número de fatores poderiam ser levantadas na busca das causas dessas diferenças. Entre estes podem estar, por exemplo, a pertinência temática dos artigos já publicados pelas revistas para com os artigos em produção, produtividade dos periódicos, longevidade, tiragem, cobertura territorial, presença e acessibilidade a coleções, indexação em base de dados, disponibilidade de artigos completos em mais de um suporte (papel e eletrônico), além de outros, dentre os quais a questão da auto-citação antes mencionada. Entretanto, é pertinente destacar o fato de que, ao não ser estabelecido um recorte cronológico quanto ao período de edição da unidade citada, é possibilitado àquela com maior longevidade receber maior número de citações, e que o inverso ocorra principalmente com periódicos de surgimento mais recente. Em outros termos, a inexistência de recorte cronológico, como aqui empregado, permite interpretar a contribuição do periódico como um todo, impactando artigos publicados em 2003.

Embora em estudos comparativos se deva padronizar o período de referência, é oportuno mencionar restrições que são feitas ao FI (COURA; WILLCOX, 2006, p. 294) pelo fato de que um período de avaliação de 2 anos representa muito pouco na área científica.

Como já foi apontado no item dos resultados, e é observável na TAB. 2, ordenamentos classificatórios que são estabelecidos com base nas variáveis $r, a, f$ ou $p$ apresentam tanto divergências entre si como em relação ao IDS. Uma causa para isso refere-se ao fato de que na 
modelagem deste índice é considerado que o fenômeno da dispersão se faz por segmentos hierarquizados de distribuição, ponderalmente diferenciados em relação a particularidades de distribuição em cada segmento. Isto é, $r, a, f$ e $p$ exercem, respectivamente, pesos sucessivamente maiores no resultado na composição do calculo do IDS. Assim, variações iguais que ocorram, por vez, a cada um destes parâmetros repercutem diversamente no valor do IDS. Essa propriedade matemática do IDS se coaduna com a idéia, por exemplo, de que uma informação encontra-se mais dispersa (física e/ou bibliologicamente) se for publicada em dois periódicos $(p=2)$ do que se for publicada em dois artigos $(a=2)$ de um mesmo fascículo.

A análise de diversas situações que determinam a ordem classificatória apresentada na TAB. 1 permite verificar que uma pequena variação de medida no nível hierárquico maior pode compensar variações maiores e de sentido oposto em uma ou mais das variáveis de menor hierarquia. De modo análogo, variações concomitantes e de mesmo sentido nestas, podem superar variações em sentido oposto na variável de hierarquia maior.

Por outro lado, verifica-se que $p$ e $f$ constituiriam parâmetros inadequados para, isoladamente, diferenciar periódicos quanto a sua dispersão. Isto se dá porque em relação a eles, diversos postos de ordenamento concentram vários periódicos. Possivelmente este aspecto contribua para que as divergências classificatórias referidas àquelas duas variáveis sejam estatisticamente significantes em relação às demais. Já a concordância entre $f$ e $L$ é compatível com a relação intrínseca entre número de fascículos, tempo de existência do periódico e correspondente probabilidade de ocorrência de citações à unidade em foco.

De outro modo, $O$ fato de se manterem correlações ordinais estatisticamente significantes tanto de $p$ e de $f$ como dos demais parâmetros com o IDS, constitui indício de que a operacionalização do mesmo constitui via de classificação que compatibiliza divergências de ordenamento estabelecidas em função dos diversos parâmetros, indiferente ao peso que estes tenham na composição do indicador.

Já a apresentação na TAB. 3 da proporção com que cada coeficiente de cada nível de distribuição participa da medida do IDS, possibilita caracterizar a natureza desse indicador. Assim, iniciando-se por caracterizar cada parcela desse índice tem-se que, o coeficiente "Ca" (nível macro de distribuição), relacionando a diversidade de suportes bibliográficos (medidas de $\mathrm{p} \mathrm{e} \mathrm{P}$ ) e o número de divisões físicas destes ( $\mathrm{f}$ e $\mathrm{F}$ ), consigna-lhe a representação de dispersão (ou espalhamento) físicobibliográfico do impacto. A relação entre o número de unidades literárias impactadas (a) e o número de divisões físicas dos periódicos (f ou F) consigna ao coeficiente $\mathrm{Ce}$ (da mesodistribuição) a representação de espalhamento físico do impacto.

O relacionamento da freqüência do impacto $(r)$ com o número de unidades literárias (a ou $\mathrm{A}$ ), consigna ao coeficiente $\mathrm{Ci}$ (nível micro de distribuição), representação que refere grau de incidência do impacto. 
Portanto, associando-se a essas representações sua participação proporcional, de acordo com a TAB. 3, verifica-se que a ínfima participação quanto ao que representa a incidência de impacto - $\mathrm{Ci}$-, possivelmente constitua um fator decisivo apenas no estabelecimento de postos classificatórios de periódicos quando estes se assemelham quanto ao somatório das demais parcelas. Esse aspecto, entretanto, não chegou a ocorrer no presente estudo. Assim, a preponderância dos fatores que representam espalhamento físico e bibliográfico do impacto constitui propriedade que confere ao IDS uma representação bivalente do fenômeno. Por sua vez, a relação quanto à distribuição de referências em artigos pode mais propriamente ser considerada como uma dispersão endógama, que, como já caracterizado, tem mínima repercussão sobre o cálculo do IDS.

Portanto, o IDS expressando o espalhamento de referências feitas ao periódico, caracteriza metricamente uma situação de visibilidade que contribui para sua continuada difusão.

\section{Considerações finais}

O que até aqui ficou exposto responde ao objetivo estabelecido para este trabalho, demonstrando a possibilidade de utilizar o índice de dispersão segmentar - IDS como ferramenta para classificar periódicos quanto ao aspecto bibliométrico que aqui representou.

De acordo com as medidas estabelecidas, que conjugam os fatores: da quantificação relativa das unidades impactadas e da sua distribuição por segmentos ou níveis, o perfil classificatório correspondente ao ano 2003 dos 14 periódicos utilizados para exemplificação ficou, segundo ordenamento decrescente, assim constituído: Revista Brasileira de Enfermagem, Revista da Escola de Enfermagem da USP, Revista Latino Americana de Enfermagem, Texto \& Contexto - Enfermagem, Acta Paulista de Enfermagem, Nursing (São Paulo), Revista de Enfermagem UERJ, Revista Gaúcha de Enfermagem, Revista Paulista de Enfermagem, Escola Anna Nery Revista de Enfermagem, Cogitare Enfermagem, REME Revista Mineira de Enfermagem, OBJN - Online Brazilian Journal of Nursing, Revista Eletrônica de Enfermagem.

Considerando os valores proporcionais referentes às parcelas que compõem o indicador, conclui-se que na medida do IDS predomina o efeito da macrodistribuição do impacto (relação entre número de periódicos e fascículos portadores do impacto), não sendo, porém, este um fator que, por si só, determina as posições classificatórias. A relação entre fascículos e artigos impactados tem menor repercussão, enquanto é ínfima a influência do fator em que intervém o número de referências ao periódico. A bivalência do IDS expressa assim um dimensionamento físicobibliográfico à representação do que, pelas propriedades evidenciadas, considera-se como apropriadamente denominado de dispersão de impacto. 


\section{Referências}

BRAUN, T.; SCHUBERT, A. Dimensions of scientometric indicator datafiles world science in 1990-1994. Scientometrics, v. 38, n. 1, p. 175-204, 1997.

COURA, J. R.; WILLCOX, L. C. B. Fator de impacto, produção científica e qualidade das revistas médicas brasileiras. Memórias do Instituto Oswaldo Cruz, v. 98, n. 3, p. 293-297, 2003.

FRANDSEN, T. F. Geographical concentration: the case of economics journals. Scientometrics, v. 63, n. 1, p. 69-85, 2005.

FROTA, M. G. C. A delimitação das unidades de análise em ciência da informação. Ciência da Informação, v. 27, n. 3, p. 262-267, 1998.

GLÄNZEL, W.; MOED, H. F. Journal impact measures in bibliometric research. Scientometrics, v. 53, n. 2, p. 171-193, 2002.

KORTELAINEN, T. A. M. Studing the international diffusion of a national scientific journal. Scientometrics, v. 51, n. 1, p.133-146, 2001.

McGRATH, W. E. The unit of analysis (objects of study) in bibliometrics and scientometrics. Scientometrics, v. 35, n. 2, p. 257-264, 1996.

PORTAL, S. G. Modelo teórico para el estudio métrico de la información documental. Gijón (Astúrias, ES): Ediciones Trea, S. L., 2005. 171 p.

PORTAL de Revistas de Enfermagem. Resultado da Busca. Disponível em $<$ http://enfermagem.bvs.br/portalrev/adv search control.php?xsl=xsl/ad $v$ search result.xsl\&lang $=\ldots$... Acesso em: 16 jun. 2006.

ROWLANDS, I. Journal diffusion factors: a new approach to measuring research influence. Aslib Proceedings, v. 54, n. 2, p. 77-84, 2002.

RUMMLER, G. Modelagem de um indicador bibliométrico para análise da dispersão de conhecimentos. Ciência da Informação, v. 35, n. 1, p. 63-71, 2006.

SEN, S. K. For what purpose are the bibliometric indicators and how should they work. Disponível em http://www.ricyt.org/interior/normalizacion/IV taller/sen.pdf Acesso em: 18 jul. 2005.

SINGLENTON, Jr. R. et al. Approaches to social research. New York: Oxford University Press, 1970 apud FROTA, M. G. C. A delimitação das unidades de análise em ciência da informação. Ciência da Informação, v. 27, n. 3, p. 262-267, 1998.

SPINAK, E. Indicadores cienciométricos. Ciência da Informação, v. 27, n. 2, p. 141-148, 1998.

SPSS INC. SPSS 9.0 for Windows. Chicago, IL: SPSS Inc., 1998.

STREHL, L. O fator de impacto do ISI e a avaliação científica: aspectos conceituais e metodológicos. Ciência da Informação, v. 34, n. 1, p. 19-27, 2005.

THOMSON SCIENTIFIC. Journal seletion process. Disponível em $<$ http://scientific. thomson.com/mjl/selection/>. Acesso em: 20 out. 2005. 
THOMSON. Thomson Scientific.The ISI Impact Factor. Disponível em: http://scientific.thomson.com/free/essays/journalcitationreports/impactfac tor. Acesso em: 21 set. 2006.

VAN RAAN, A. F. J. Measuring science. In: MOED, H. F.; GLÄNZEL, W.; $\mathrm{SCHMOCH}, \mathrm{U}$. (Eds.). Handbook of quantitative science and technology research. Dordrecht: Kluver Academic Publishers, 2004. p. 19-50.

VINKLER, P. An attempt of surveying and classifying bibliometric indicators for scientometric purposes. Scientometrics, v. 13, n. 5-6, p. 239-259, 1998.

VINKLER, P. Relations of relative scientometric indicators. Scientometrics, v. 58, n. 3, p. 687-694, 2003. 